\title{
OBSERVATIONAL EVIDENCE OF THE FORMATION OF CYANOPOLYYNES IN CRL 618 THROUGH THE POLYMERIZATION OF HCN
}

\author{
Juan R. Pardo, José Cernicharo, and Javier R. Goicoechea ${ }^{1}$ \\ Departamento de Astrofísica Molecular e Infrarroja, Instituto Estructura de la Materia, CSIC, Serrano 121, E-28006 Madrid, Spain; \\ pardo@damir.iem.csic.es, cerni@damir.iem.csic.es, javier@damir.iem.csic.es \\ Received 2005 January 18; accepted 2005 April 6
}

\begin{abstract}
The abundance ratio of consecutive members of the cyanopolyyne family has been explored in CRL 618 using data acquired in a complete line survey covering the frequency range $81-356 \mathrm{GHz}$. The $J_{\text {up }}$ range explored for the different molecules is $1-4$ for $\mathrm{HCN}$ and $\mathrm{HNC}, 9-39$ for $\mathrm{HC}_{3} \mathrm{~N}, 31-133$ for $\mathrm{HC}_{5} \mathrm{~N}$, and $72-85$ for $\mathrm{HC}_{7} \mathrm{~N}$ (not detected beyond $\left.J_{\text {up }}=85\right)$. The lowest vibrationally excited state of $\mathrm{HC}_{7} \mathrm{~N}\left(\nu_{15}\right.$ at $\left.62 \mathrm{~cm}^{-1}\right)$ has been tentatively detected. Data analysis has been performed by extending our previous geometric and radiative transfer model of the slowly expanding envelope (SEE) surrounding the compact central continuum source of CRL 618, which was established from the study of rotational lines in several vibrationally excited states of $\mathrm{HC}_{3} \mathrm{~N}$. The new lines analyzed here require modeling of the high-velocity wind (HVW) component and the colder circumstellar gas, a remnant of the AGB phase of CRL 618. The derived $\mathrm{HC}_{3} \mathrm{~N} / \mathrm{HC}_{5} \mathrm{~N}$ and $\mathrm{HC}_{5} \mathrm{~N} / \mathrm{HC}_{7} \mathrm{~N}$ abundance ratios from this set of uniformly calibrated lines are between $\sim 3$ and 6 in the different regions, similar to standard values in the circumstellar and interstellar media and consistent with previous estimates obtained from ISO observations and chemical models. However, the abundance ratios of $\mathrm{HC}_{3} \mathrm{~N}, \mathrm{HC}_{5} \mathrm{~N}$, and $\mathrm{HC}_{7} \mathrm{~N}$ with respect to $\mathrm{HCN}$ are at least 2 orders of magnitude larger than those typical for AGB C-rich stars, such as IRC +10216 . This fact indicates that in the short transition toward the planetary nebula phase, $\mathrm{HCN}$ is quickly reprocessed into longer cyanopolyyne chains. A similar behavior was previously found in this object for the polyacetylenic chains $\left(\mathrm{C}_{2 n} \mathrm{H}_{2}\right)$.
\end{abstract}

Subject headings: astrochemistry — circumstellar matter — ISM: molecules — radio lines: stars — stars: AGB and post-AGB - stars: carbon — stars: individual (CRL 618)

\section{INTRODUCTION}

CRL 618 is a C-rich protoplanetary nebula (PPN). Its chemical richness and its complex morphology, with a central B0 star surrounded by an ultracompact $\mathrm{H}$ II region and a thick molecular and dusty envelope (Bujarrabal et al. 1988) with different outflows (Cernicharo et al. 1989, hereafter C89), are now well known thanks to many detailed observational studies. Detailed chemical models for this object have been developed (Cernicharo 2004), indicating the rapid evolution of the central star and its influence on the circumstellar ejected material. Changes seem to occur at short - almost human - timescales. The most recent observational results on the morphology are the interferometric observations presented in Sánchez-Contreras \& Sahai (2004). Concerning the chemical composition, a line survey from 80 to $275 \mathrm{GHz}$ has been recently completed (J. Cernicharo et al. 2005, in preparation).

After our study of the physical conditions in the slowly expanding envelope (SEE) around the ultracompact $\mathrm{H}$ II region of CRL 618 using vibrationally excited states of $\mathrm{HC}_{3} \mathrm{~N}$ (Pardo et al. 2004, hereafter P04), we focus now on the entire cyanopolyyne family: $\mathrm{HCN}, \mathrm{HNC}, \mathrm{HC}_{3} \mathrm{~N}, \mathrm{HC}_{5} \mathrm{~N}$, and $\mathrm{HC}_{7} \mathrm{~N}$ (longer members have not been detected in our survey).

$\mathrm{HC}_{5} \mathrm{~N}$ is the second molecule, after $\mathrm{HC}_{3} \mathrm{~N}$, in terms of total number of lines detected in the millimeter spectrum of CRL 618. It is a linear molecule with a rotational constant of $1331.330 \mathrm{MHz}$ and a dipole moment of 4.33 D, first discovered in space by Little et al. (1978). The two lowest energy doubly degenerate bending modes $\left(\nu_{11}\right.$ and $\left.\nu_{10}\right)$ have energies of 105 and $230 \mathrm{~cm}^{-1}$, respectively (Yamada et al. 2004). On the other

\footnotetext{
${ }^{1}$ Current address: LERMA-École Normale Supérieure (UMR 8112 du CNRS), 24 Rue Lhomond, 75231 Paris, France.
}

hand, $\mathrm{HC}_{7} \mathrm{~N}$ was first discovered in space by Kroto et al. (1978). Its rotational constant and dipole moment are $564.0011 \mathrm{MHz}$ and $4.82 \mathrm{D}$, respectively. $\mathrm{HC}_{3} \mathrm{~N} / \mathrm{HC}_{5} \mathrm{~N}$ and $\mathrm{HC}_{5} \mathrm{~N} / \mathrm{HC}_{7} \mathrm{~N}$ abundance ratios between 1.4 and 3.0 were found in molecular clouds since the earliest studies (Snell et al. 1981). Finally, HCN, with a dipole moment and rotational constant of $2.98 \mathrm{D}$ and $44315.976 \mathrm{MHz}$, respectively, is one of the most abundant molecules in the interstellar and circumstellar media. In many cases, HCN lines are optically thick, so that observations of isotopologues or vibrationally excited states are preferred to probe molecular clouds and circumstellar envelopes. The other two molecules discussed in this paper have the following dipole moments and rotational constants: $3.73 \mathrm{D}$ and $4549.059 \mathrm{MHz}\left(\mathrm{HC}_{3} \mathrm{~N}\right)$ and $2.98 \mathrm{D}$ and $45331.7845 \mathrm{MHz}$ (HNC).

The goal of the study conducted in this paper is to get abundance ratios of the different members of the cyanopolyyne family in CRL 618 and to compare them with those observed in prototypical asymptotic giant branch (AGB) objects such as IRC +10216 . In addition, by using new polar species we aim at improving and extending our previous model of CRL 618 (see P04). This is another step toward a final model for the whole millimeter-wave spectrum of this source. The observational procedure and SEE model developments were described in detail in P04; here we only give a short summary in $\S \S 2$ and 3 . The analysis of the $\mathrm{HCN}, \mathrm{HNC}, v=0 \mathrm{HC}_{3} \mathrm{~N}, \mathrm{HC}_{5} \mathrm{~N}$, and $\mathrm{HC}_{7} \mathrm{~N}$ data, with the extension of the model, is presented in $\S 4$. Comparisons of the results with chemical model predictions and other discussions are presented in $\S 5$. The work is summarized in $\S 6$.

\section{OBSERVATIONS}

The observations presented in this paper are part of two line surveys of CRL 618. The first one, now complete, was carried 
TABLE 1

Observational Parameters of Selected $\mathrm{HC}_{5} \mathrm{~N}$ Lines in CRL 618

\begin{tabular}{|c|c|c|c|c|c|c|c|c|}
\hline \multirow[b]{2}{*}{$J_{\text {up }}$} & \multirow[b]{2}{*}{$v$ STATE } & \multirow[b]{2}{*}{$\begin{array}{c}\nu \\
(\mathrm{GHz})\end{array}$} & \multicolumn{3}{|c|}{ ABSORPTION } & \multicolumn{3}{|c|}{ EMISSION } \\
\hline & & & $\begin{array}{c}v \\
\left(\mathrm{~km} \mathrm{~s}^{-1}\right)\end{array}$ & $\begin{array}{c}\Delta v \\
\left(\mathrm{~km} \mathrm{~s}^{-1}\right)\end{array}$ & $\begin{array}{c}A \\
\left(\mathrm{~K} \mathrm{~km} \mathrm{~s}^{-1}\right)\end{array}$ & $\begin{array}{c}v \\
\left(\mathrm{~km} \mathrm{~s}^{-1}\right)\end{array}$ & $\begin{array}{c}\Delta v \\
\left(\mathrm{~km} \mathrm{~s}^{-1}\right)\end{array}$ & $\begin{array}{c}A \\
\left(\mathrm{~K} \mathrm{~km} \mathrm{~s}^{-1}\right)\end{array}$ \\
\hline \multirow[t]{10}{*}{$33 \ldots \ldots \ldots$} & $v=0$ & 87.86363 & -20.3 & 18.9 & 2.7 & -43.3 & 23.3 & -0.87 \\
\hline & $\nu_{11} 1^{-}$ & 88.00874 & -20.4 & 10.0 & 0.29 & -33.1 & 8.6 & -0.29 \\
\hline & $\nu_{11} 1^{+}$ & 88.08534 & -22.6 & 23.2 & 0.54 & -34.7 & 14.1 & -0.55 \\
\hline & $2 \nu_{11} 0$ & 88.22163 & -21.1 & 6.8 & 0.09 & -30.5 & 16.9 & -0.17 \\
\hline & $2 \nu_{11} 2^{-}$ & 88.23055 & -24.8 & - & - & -31.2 & - & - \\
\hline & $2 \nu_{11} 2^{+}$ & 88.24034 & -23.2 & 5.1 & 0.09 & -33.5 & 5.9 & -0.08 \\
\hline & $\nu_{10} 1^{+}$ & 88.04187 & & & & -34.7 & 6.7 & -0.05 \\
\hline & $3 \nu_{11} 1^{-}$ & 88.33509 & & & & -31.2 & 4.9 & -0.05 \\
\hline & $3 \nu_{11} 3^{+}$ & 88.41807 & & & & -28.5 & 7.2 & -0.16 \\
\hline & $3 \nu_{11} 1^{+}$ & 88.48921 & & & & -31.4 & 7.2 & -0.12 \\
\hline $34 \ldots \ldots \ldots \ldots$ & $v=0$ & 90.52589 & -21.1 & 19.1 & 4.0 & -38.4 & 34.1 & -1.9 \\
\hline \multirow[t]{4}{*}{ 35...................... } & $v=0$ & 93.18813 & -21.1 & 19.3 & 4.2 & -37.3 & 32.7 & -2.6 \\
\hline & $\nu_{11} 1^{-}$ & 93.34201 & -22.5 & 8.4 & 0.49 & -33.0 & 18.7 & -0.89 \\
\hline & $\nu_{11} 1^{+}$ & 93.42323 & -23.2 & 22.2 & 0.88 & -33.5 & 12.9 & -0.69 \\
\hline & $2 \nu_{11} 0$ & 93.56650 & -21.7 & & & -32.0 & 8.2 & -0.30 \\
\hline $52 \ldots \ldots \ldots \ldots \ldots$ & $v=0$ & 138.44167 & $-21.3^{*}$ & 19.8 & 3.6 & $-38.0^{*}$ & 17.7 & -1.3 \\
\hline 58...................... & $v=0$ & 154.41110 & $-21.3^{*}$ & 14.8 & 2.8 & $-38.0^{*}$ & 20.8 & -1.2 \\
\hline \multirow[t]{6}{*}{59} & $v=0$ & 157.07253 & $-21.3^{*}$ & 16.1 & 2.9 & $-38.0^{*}$ & 19.9 & -1.2 \\
\hline & $\nu_{11} 1^{-}$ & 157.33140 & -23.8 & 13.3 & 1.6 & -28.3 & 17.6 & -1.2 \\
\hline & $\nu_{11} 1^{+}$ & 157.46775 & -23.7 & 14.5 & 0.92 & -31.8 & 8.6 & -0.47 \\
\hline & $2 \nu_{11} 0$ & 157.67636 & -19.1 & 16.6 & 0.43 & -29.9 & 6.8 & -0.19 \\
\hline & $2 \nu_{11} 2^{-}$ & 157.72678 & -21.0 & 14.0 & 0.47 & -31.2 & 9.7 & -0.28 \\
\hline & $\nu_{10} 1^{+}$ & 157.39079 & -20.0 & 13.2 & 0.32 & & & \\
\hline \multirow[t]{2}{*}{$96 \ldots \ldots \ldots \ldots$} & $v=0$ & 255.50940 & -24.6 & 10.1 & 0.94 & & & \\
\hline & $\nu_{11} 1^{-}$ & 255.92839 & -23.6 & 6.1 & 0.27 & & & \\
\hline 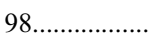 & $v=0$ & 260.82794 & -21.6 & 10.9 & 0.88 & & & \\
\hline
\end{tabular}

NotES.- Velocity centroid, width, and integrated area obtained from Gaussian fits of the absorption and emission components. See beginning of $\S 4$ for more details.

out with the Institut de Radioastronomie Millimétrique (IRAM) $30 \mathrm{~m}$ telescope from 1994 to 2002 (J. Cernicharo et al. 2005, in preparation) with a frequency coverage of $81-279 \mathrm{GHz}$, except for frequencies with high atmospheric opacity around 119 and $183 \mathrm{GHz}$. The second one, still ongoing but complete for the species we focus on in this work, is performed with the Caltech Submillimeter Observatory (CSO) telescope between 280 and $356 \mathrm{GHz}$. For both instruments the frequency resolution used has been around $1 \mathrm{MHz}$ with $\sim 0.5 \mathrm{GHz}$ wide spectrometers. The $J_{\text {up }}$ range covered for rotational transitions is $1-4$ for $\mathrm{HCN}$ and $\mathrm{HNC}, 9-39$ for $\mathrm{HC}_{3} \mathrm{~N}, 31-133$ for $\mathrm{HC}_{5} \mathrm{~N}$, and $71-316$ for $\mathrm{HC}_{7} \mathrm{~N}$, although the lines are not detected beyond $J_{\text {up }}=85$. See P04 for details on the observational procedures at the two facilities.

\section{PREVIOUS MODEL SUMMARY}

A model has been developed to study the physical conditions that explain the whole observed millimeter-wave spectrum of CRL 618. Precise knowledge of the spectral behavior of the continuum emission (see P04) is paramount, since it plays a very important role in the observed line profiles (especially in the emission-to-absorption line ratio). The model consists of a gas envelope around a central continuum source, considered to be spherical with size and effective temperature adjusted to fit the continuum IRAM $30 \mathrm{~m}$ data (P04) and assumed to keep the same spectral index in the range $280-356 \mathrm{GHz}$ (CSO observations). The description of the gas component allows different shells with different physical conditions to be included in the model. The geometry is not restricted to be spherical. Elongated shells with both radial and azimuthal velocity components can be defined.
Since the observations reveal that different gas regions are traced by different molecular species, we proceed in our analysis in different steps. The first one has been to study those species arising from the inner $\sim 1 " .5 \mathrm{SEE}$, with outflow velocities of $\sim 10-15 \mathrm{~km} \mathrm{~s}^{-1}$, turbulence velocities around $3.5 \mathrm{~km} \mathrm{~s}^{-1}$, and temperatures in the range $250-275 \mathrm{~K}$. The full description of the SEE model is given in P04. The extension of the SEE model to include both the colder, outer circumstellar gas (necessary to explain the rotational lines in the ground vibrational state of $\mathrm{HC}_{3} \mathrm{~N}, \mathrm{HC}_{5} \mathrm{~N}$, and $\left.\mathrm{HC}_{7} \mathrm{~N}\right)$ and the high-velocity wind component (seen in lines of some abundant species, including $\left.v=0 \mathrm{HC}_{3} \mathrm{~N}\right)$ is one of the targets of this work. This is discussed in detail in $\S 4$.

\section{ANALYSIS AND DISCUSSION}

The first step of the analysis has been to perform Gaussian fits of selected $\mathrm{HC}_{5} \mathrm{~N}$ lines in the 3,2 , and $1.3 \mathrm{~mm}$ windows. A summary for a set of lines representative of the different vibrational states and frequency ranges is presented in Table 1, where the areas are expressed in $\mathrm{K} \mathrm{km} \mathrm{s}^{-1}\left(T_{A}^{*} v_{\mathrm{LSR}}\right)$, parameters that could not be determined are marked with a dash, and parameters that had to be fixed in order to obtain a reasonable fit are marked with asterisks. In addition, when the velocity, width, and area of one component are left blank in the table it means that the component is not detected in the particular transition. These observational results already reveal an interesting behavior that is crucial for the analysis: lines in the $v=0$ state have absorption and emission components that are in general $15-25 \mathrm{~km} \mathrm{~s}^{-1}$ wide at 3 and $2 \mathrm{~mm}$, with the absorption centered 


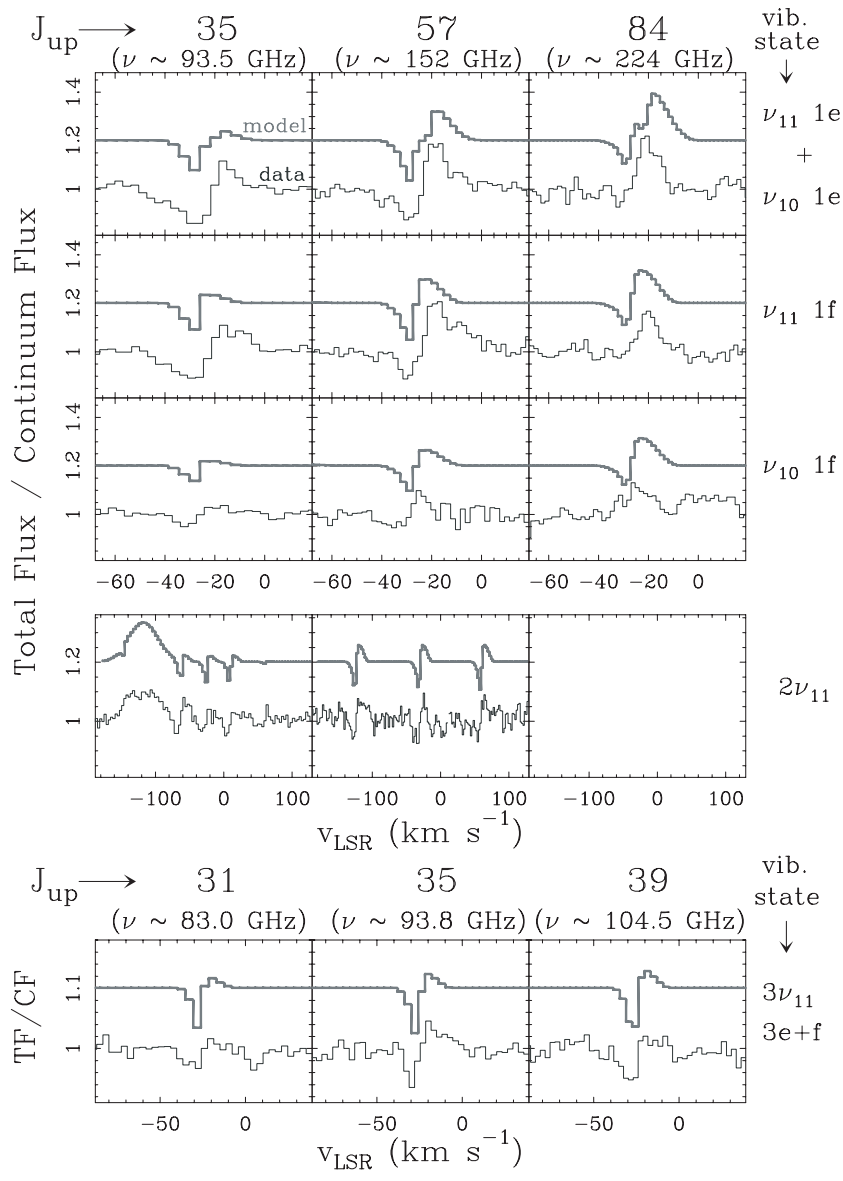

FIG. 1.-Sample of detected lines in vibrationally excited states of $\mathrm{HC}_{5} \mathrm{~N}$ toward CRL 618. Model results, shifted by $0.2 F_{c}$ ( $F_{c}$ is continuum flux) for all vibrational states except for $3 \nu_{11}\left(0.1 F_{c}\right.$ shift $)$, are shown at the velocity resolution of the data ( $\Delta v=c \Delta \nu / \nu$, with $\Delta \nu \simeq 1 \mathrm{MHz}$ for all spectra) for a $\mathrm{HC}_{3} \mathrm{~N} /$ $\mathrm{HC}_{5} \mathrm{~N}$ value of 3 . All other physical conditions are as determined by $\mathrm{P} 04$ in the SEE (the only region considered). The extra feature that appears in the $2 \nu_{11}$ $J_{\text {up }}=35$ spectrum is a blending of the $\beta 51$ recombination line of atomic hydrogen plus the $J=83-82$ line of $v=0 \mathrm{HC}_{7} \mathrm{~N}$.

at around -37 to $-42 \mathrm{~km} \mathrm{~s}^{-1}$, whereas lines in vibrationally excited states, in the same spectral region, appear narrower $\left(\sim 7-15 \mathrm{~km} \mathrm{~s}^{-1}\right)$ with the absorption component (sometimes the only one detected) centered at approximately -28 to $-33 \mathrm{~km} \mathrm{~s}^{-1}$. These differences tend to disappear as frequency increases, and the line profiles in the $v=0$ state are dominated by the emission component only. These facts suggest that the rotational lines in the ground state of $\mathrm{HC}_{5} \mathrm{~N}$ are mostly formed in a region with quite different physical conditions than the one responsible for the lines in vibrationally excited states. This is carefully explored in this section.

\subsection{Vibrationally Excited $\mathrm{HC}_{5} \mathrm{~N}$}

The latest laboratory data on the $\nu_{11}$ and $\nu_{10}$ vibrational states of $\mathrm{HC}_{5} \mathrm{~N}$, their overtones and their combinations, have been presented by Yamada et al. (2004). In our line survey, we have detected rotational lines in the following vibrationally excited states of $\mathrm{HC}_{5} \mathrm{~N}$ (most of them for the first time in space): $\nu_{11}$, $2 \nu_{11}, \nu_{10}$, and $3 \nu_{11}$ at energies of $105,210,230$, and $315 \mathrm{~cm}^{-1}$, respectively. Since these lines also should arise from the SEE analyzed in $\mathrm{P} 04$, we have just tried to find the $\mathrm{HC}_{3} \mathrm{~N} / \mathrm{HC}_{5} \mathrm{~N}$ abundance ratio that best fits the observations, keeping the same $\mathrm{SEE}$ physical parameters as in $\mathrm{P} 04$. The result is $\mathrm{HC}_{3} \mathrm{~N} / \mathrm{HC}_{5} \mathrm{~N} \sim$ $3 \pm 1$ (see Fig. 1). This result confirms previous estimates made

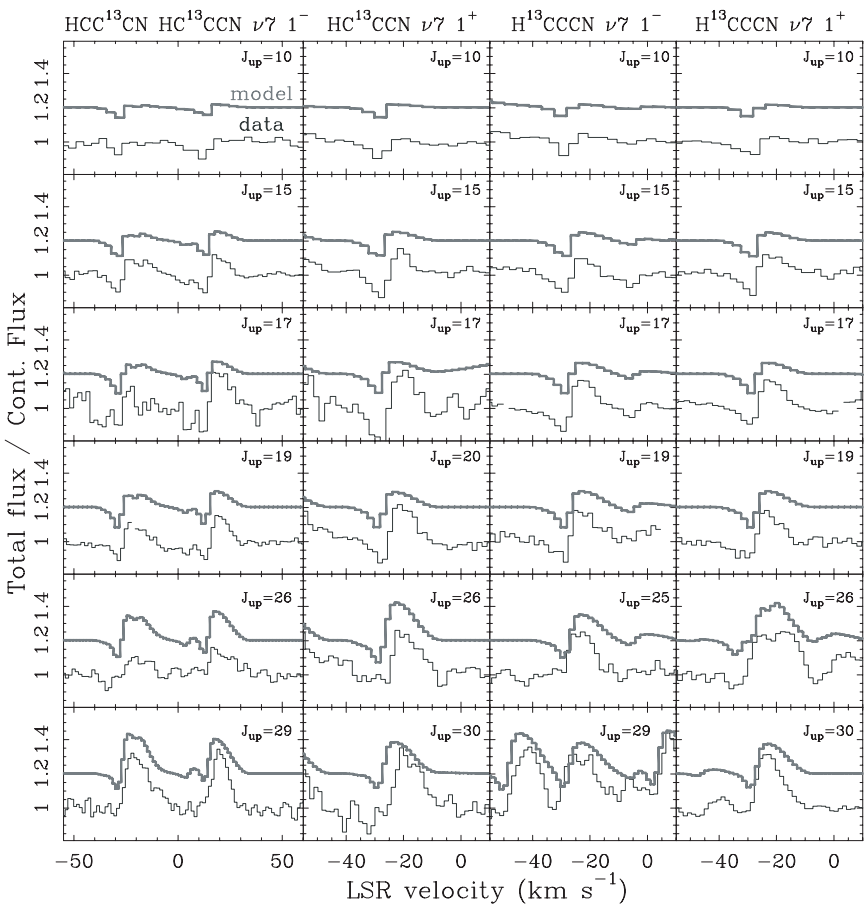

FIG. 2.-Model predictions considering a ${ }^{12} \mathrm{C} /{ }^{13} \mathrm{C}$ isotopic ratio of 15 in the SEE of CRL 618 (shifted by $0.2 F_{c}$ ) compared to the observations for a sample of lines belonging to single- ${ }^{13} \mathrm{C}$-substituted $\mathrm{HC}_{3} \mathrm{~N}$ in the $\nu_{7}$ state. The velocity resolutions of the model and data are the same $(\Delta v=c \Delta \nu / \nu$, with $\Delta \nu \simeq 1$ $\mathrm{MHz}$ for all spectra and $\nu \simeq J_{\text {up }} 9.1 \mathrm{GHz}$ ). Two weak features, between the $\mathrm{HCC}^{13} \mathrm{CN}$ and $\mathrm{HC}^{13} \mathrm{CCN} \nu_{7} 1^{-}$lines, clearly seen in the spectra with the best signal-to-noise ratio, correspond to the $\nu_{6} 1^{+}$lines of both species. They have also been included in the model. All other extra features that appear in some spectra belong to vibrationally excited states of $\mathrm{HC}_{3} \mathrm{~N}$.

from vibrational bands of both species seen in absorption by the Infrared Space Observatory (ISO) around $15 \mu \mathrm{m}$ (Cernicharo et al. 2001). Obviously, the fit cannot be perfect for so many lines. In particular, Figure 1 shows that some absorption exists between -50 and $-40 \mathrm{~km} \mathrm{~s}^{-1}$ for $J_{\text {up }}=35$ in the $\nu_{11}$ state that is not reproduced by the model. This could be due to a fraction of a higher velocity outflow that intersects the line of sight toward the continuum source that has not been considered in the model. This absorption component is otherwise clearly visible in the rotational lines inside the $\nu_{7}$ state of $\mathrm{HC}_{3} \mathrm{~N}$ but does not affect the other lines shown in Figure 1. The detections in the $\mathrm{HC}_{5} \mathrm{~N}$ vibrational states extend from $J_{\text {up }}=31$ to $J_{\text {up }}=104$ for $\nu_{11}$, $J_{\text {up }} \sim 87$ for $2 \nu_{11}, J_{\text {up }} \sim 77$ for $\nu_{10}$, and $J_{\text {up }} \sim 53$ for $3 \nu_{11}$. The fact that for $\mathrm{HC}_{3} \mathrm{~N}$ we detect rotational lines from vibrational states a factor of 3-4 higher in energy than for $\mathrm{HC}_{5} \mathrm{~N}$ is consistent, for the sensitivity of the survey, with the derived $\mathrm{HC}_{3} \mathrm{~N} / \mathrm{HC}_{5} \mathrm{~N}$ abundance ratio and the dipole moment and partition function ratios of both species.

\section{2. ${ }^{12} \mathrm{C} /{ }^{13} \mathrm{C}$ Ratio in the SEE}

The ${ }^{12} \mathrm{C} /{ }^{13} \mathrm{C}$ isotopic ratio in the SEE can be derived using $\mathrm{HCC}^{13} \mathrm{CN}, \mathrm{HC}{ }^{13} \mathrm{CCN}$, and $\mathrm{H}^{13} \mathrm{CCCN}$ in the $\nu_{7}$ state. The $\nu_{6}$ state is also detected, but the signal-to-noise ratio is too low for any estimation. Figure 2 shows the results of the SEE model for ${ }^{12} \mathrm{C} /{ }^{13} \mathrm{C}=15$ (P04). We estimate an error bar for this value of approximately \pm 2 . Kahane et al. (1992) gave lower limits for this ratio of 18 and 3.2 depending on which transition $(J=1-0$ or $J=2-1)$ of both ${ }^{12} \mathrm{CO}$ and ${ }^{13} \mathrm{CO}$ was used. Our estimate gives a much better constraint, restricted to the SEE region, because it relies on tens of lines without the saturation problems of $\mathrm{CO}$. 


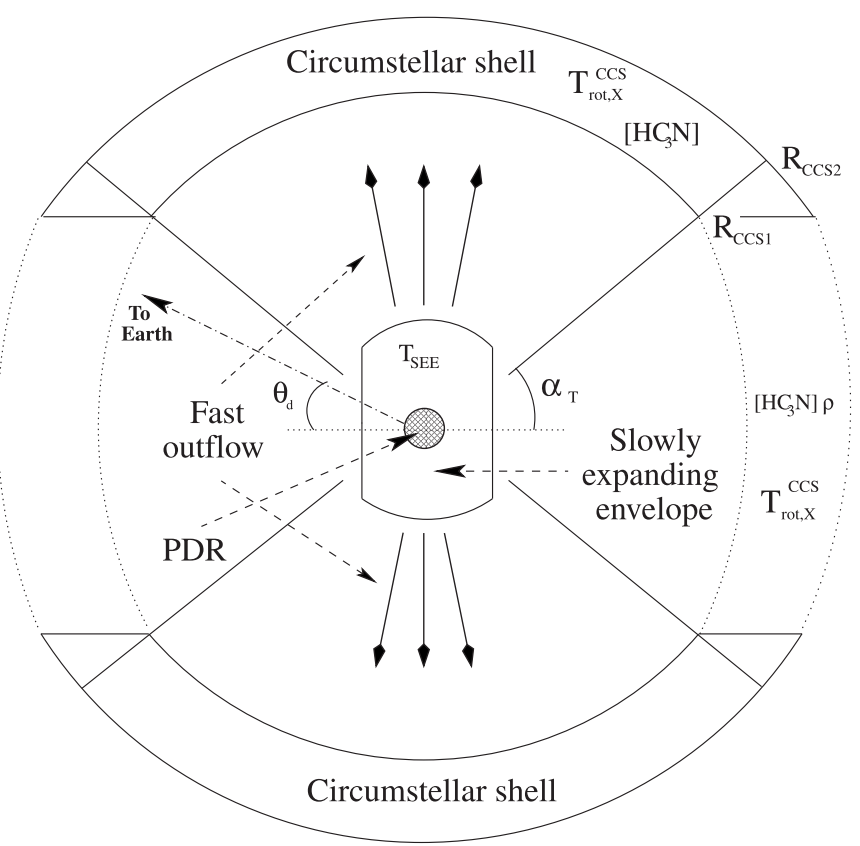

FIG. 3.-Model of CRL 618 including a cold circumstellar shell first introduced in our work to reproduce the observed rotational lines of $\mathrm{HC}_{5} \mathrm{~N}$ in its ground vibrational state. Details are in $\S 4.3$.

\subsection{Ground-State $\mathrm{HC}_{5} \mathrm{~N}$}

Using the result found in $\S 4.1$, we have run the model for the detected rotational lines of $\mathrm{HC}_{5} \mathrm{~N}$ in its ground vibrational state. From these calculations it becomes clear that the SEE alone cannot account for these lines. A significant contribution from gas at lower temperatures must exist. In the individual lines, there is no significant evidence of the high-velocity wind (HVW) seen in the most abundant molecules (see below). Therefore, we have expanded the model presented in P04 to include a cold circumstellar shell (CCS), created during the AGB phase (see Fig. 3). We have not simply considered a spherical geometry for this cold component, in order to account for the optical/infrared images of the circumstellar envelope that show that the envelope has a bipolar structure. This was also pointed out for the molecular gas in C89 and Neri et al. (1992). The parameters that define the CCS in our model are the following:

1. $R_{\mathrm{CCS} 1}$ and $R_{\mathrm{CCS} 2}$ are the inner and outer boundaries of the shell (angles in arcseconds).

2. $\alpha_{T}$ is the truncation angle (degrees), used to take into account the fact that this shell needs to be divided into at least two zones, because the molecular density is probably larger in the polar regions along the axis of the HVW. This fact is revealed by the ratio between emission and absorption in the profiles of the analyzed $v=0 \mathrm{HC}_{5} \mathrm{~N}$ rotational lines. CCS.

3. $T_{\text {rot } \mathrm{X}}^{\mathrm{CCS}}$ is the rotational temperature $(\mathrm{K})$ for species $\mathrm{X}$ in the

4. $\left[\mathrm{HC}_{3} \mathrm{~N}\right]$ (at $R=R_{\mathrm{CCS} 1}$ ) is the number density of $\mathrm{HC}_{3} \mathrm{~N}$ at the inner part of the shell for $\alpha>\alpha_{T}$ (for $\alpha<\alpha_{T}$, the density will by multiplied by a factor $\left.\rho_{\mathrm{CCS}}\right) . \mathrm{HC}_{3} \mathrm{~N}$ is always our reference molecule for the models of CRL 618. In each region we consider a constant ratio with respect to it for each molecule.

5. $d_{\mathrm{CCS}}$ determines the radial distribution of the $\mathrm{HC}_{3} \mathrm{~N}$ abundance, which is considered to vary as $\left[\mathrm{HC}_{3} \mathrm{~N}_{R}\right]=$ $\left[\mathrm{HC}_{3} \mathrm{~N}_{R_{\mathrm{CCS} 1}}\right]\left(R / R_{\mathrm{CCS} 1}\right)^{d_{\mathrm{CCS}}}$ in the CCS.

6. $v_{\mathrm{CCS}}$ and $v_{\text {turb,CCS }}$ are the radial and turbulence velocities of the gas in the CCS.
Just as we did in P04 for the physical parameters of the SEE, we had to run a considerable number of models to find a good fit of the ground-state $\mathrm{HC}_{5} \mathrm{~N}$ lines. Again, the key parameters are the number density, the temperature, and the position of the CCS. The position has been fixed between diameters 3".0 and 4 ."5 after trying several other positions in our models. Then, the temperature and density profiles can be established by running a grid of models. All the other parameters are then explored to fine tune the shape of the line as $J_{\text {up }}$ varies. For example, some of the most remarkable differences in the line profiles from ground to vibrationally excited $\mathrm{HC}_{5} \mathrm{~N}$ are the position of the maximum depth of the absorption, its width, and the absorption/ emission ratio. As shown in Figure 1 and Table 1, the maximum depth of the absorption for vibrationally excited $\mathrm{HC}_{5} \mathrm{~N}$ happens at approximately $-30 \mathrm{~km} \mathrm{~s}^{-1}$ in the 2 and $3 \mathrm{~mm}$ windows (as for the lines in vibrationally excited states of $\mathrm{HC}_{3} \mathrm{~N}$ presented in P04), whereas for the lines in the vibrational ground state, it happens at around $-40 \mathrm{~km} \mathrm{~s}^{-1}$ and is generally wider and less deep. The observational differences have allowed us to determine the kinematic conditions in the CCS. The parameters of the best simulation (Fig. 4) are shown in Table 2. The rotational temperature is in the range $50-70 \mathrm{~K}$. In fact, a simulation with $T_{\text {rot }}=50 \mathrm{~K}$ provides a better fit for lines below $J_{\text {up }} \sim 55$, whereas $T_{\text {rot }}=70 \mathrm{~K}$ provides a better fit for the range $J_{\text {up }} \sim 55-80$. The simulation shown in Figure 4 is a compromise between the two cases $\left(T_{\text {rot }}=60 \mathrm{~K}\right)$. Above $J_{\text {up }} \sim 80$, the line profiles are almost exclusively determined by the SEE region. The error bar for the $\left[\mathrm{HC}_{3} \mathrm{~N}\right] /\left[\mathrm{HC}_{5} \mathrm{~N}\right]=3$ ratio in the $\mathrm{CCS}$ can again be estimated to be \pm 1 . The same absorption at $v_{\mathrm{LSR}}<-40 \mathrm{~km} \mathrm{~s}^{-1}$ reported in $\S 4.1$, and not perfectly modeled here, is seen in some $v=0 \mathrm{HC}_{5} \mathrm{~N}$ spectra. Absorptions centered at approximately $-40 \mathrm{~km} \mathrm{~s}^{-1}$ have previously been reported in this object in molecules such as $\mathrm{CO}$ (Herpin et al. 2002), HCN (C89 and Neri et al. 1992), $\mathrm{HCO}^{+}$ (C89; Sánchez-Contreras \& Sahai 2004), $\mathrm{HC}_{3} \mathrm{~N}$ (C89, P04), and $\mathrm{CS}$ (Hajian et al. 1995). There is general agreement to assign that absorption to the AGB circumstellar envelope.

Sánchez-Contreras \& Sahai (2004) suggest that an elongated structure of $\sim 11^{\prime \prime}$ diameter with expansion velocity of $\sim 17.5 \mathrm{~km} \mathrm{~s}^{-1}$ is responsible for part of the emission of the lowvelocity molecular gas in their $\mathrm{HCN} J=1-0$ and $\mathrm{HCO}^{+} J=$ $1-0$ interferometric maps. This structure is most probably an extension (due to a larger abundance of these molecules in cold regions) of the CCS we are modeling here for single-antenna data. The difference between this larger shell and our CCS is probably due to the different excitation conditions of the low- $J$ lines of $\mathrm{HCN}$ and $\mathrm{HCO}^{+}$and the high- $J$ lines of $\mathrm{HC}_{5} \mathrm{~N}$ explored in this work.

On the basis of the extended model, which now includes two gas regions, we can check for the presence of longer cyanopolyynes rather easily, because only their abundance ratio with respect to $\mathrm{HC}_{5} \mathrm{~N}$ or $\mathrm{HC}_{3} \mathrm{~N}$ is needed to make predictions of their lines and then check against the data (assuming the same rotational temperature). This is presented in the following subsections.

\subsection{Isotopic $\mathrm{HC}_{5} \mathrm{~N}$ and ${ }^{12} \mathrm{C} /{ }^{13} \mathrm{C}$ Ratio in the CCS}

We have looked at the possible detection of the ${ }^{13} \mathrm{C}$-substituted species of $\mathrm{HC}_{5} \mathrm{~N}$ by comparing the observations at the frequencies of their rotational lines in the ground vibrational state with the extended model results considering a ${ }^{12} \mathrm{C} /{ }^{13} \mathrm{C}$ isotopic ratio of 15 in the SEE (as derived in $\S 4.2$ ) and also in the CCS. The simulations indicate that this value is an upper limit for the CCS. This is important to later evaluate the $\mathrm{HCN}$ and $\mathrm{HNC}$ abundances from $\mathrm{H}^{13} \mathrm{CN}$ and ${ }^{13} \mathrm{C}(\S 4.6)$. 

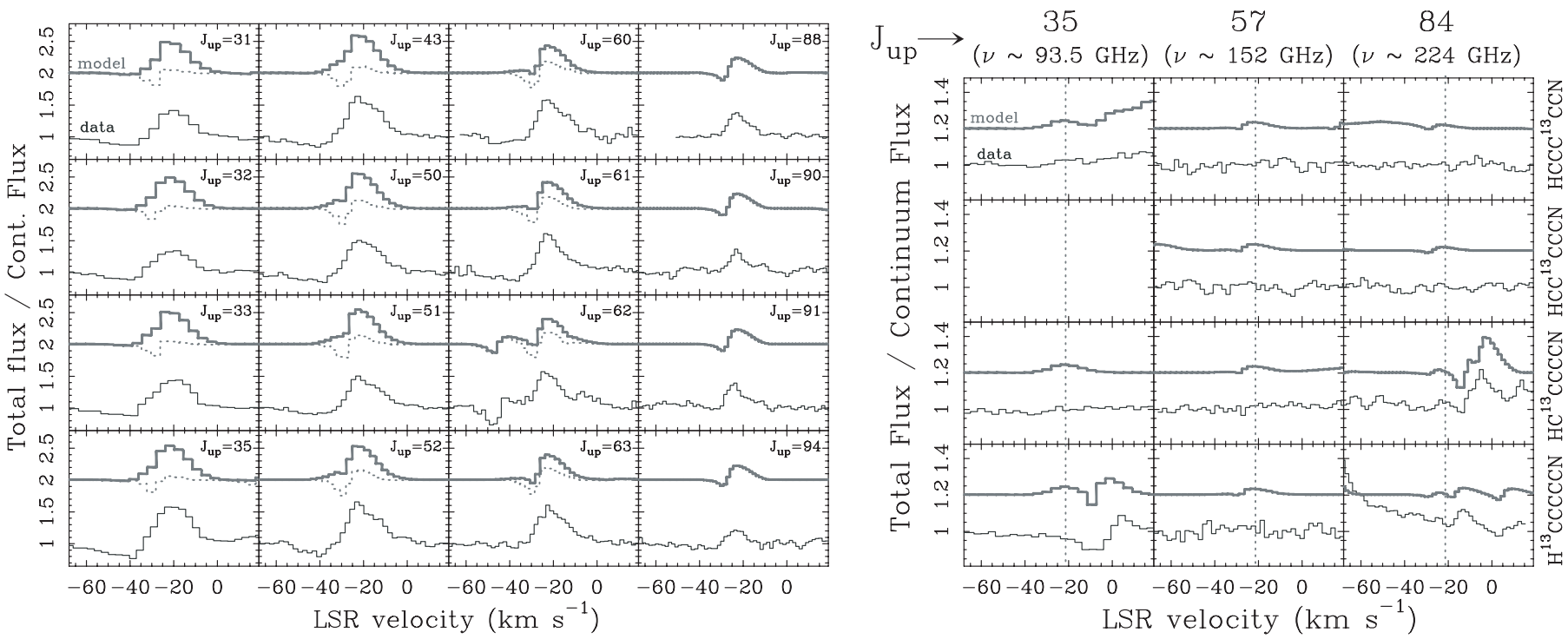

FIG. 4. - Left: Observed rotational lines of $\mathrm{HC}_{5} \mathrm{~N}$ in the ground vibrational state compared with the SEE model assuming a $\mathrm{HC}_{3} \mathrm{~N} / \mathrm{HC}_{5} \mathrm{~N}$ value of 3 (dotted histogram, shifted by $1.0 F_{c}$ ) and with the expanded model (solid histogram, shifted by $1.0 F_{c}$ ), which includes the CCS, presented in this paper ( $(4$, Fig. 3, and Table 2). Both models are basically coincident for $J_{\text {up }}$ above $\sim 80$. Right: Results of the CRL 618 model including the SEE and the CCS regions (solid histogram, shifted by $0.2 F_{c}$ ) for a sample of lines of ${ }^{13} \mathrm{C}$-substituted $\mathrm{HC}_{5} \mathrm{~N}$, compared to the observations. The simulations consider a ${ }^{12} \mathrm{C} /{ }^{13} \mathrm{C}$ value of 15 in both regions. Velocity resolutions of the data and model are the same for each plot, as explained in previous figures.

\section{5. $\mathrm{HC}_{3} \mathrm{~N}$ in the $C C S$}

The $\mathrm{HC}_{3} \mathrm{~N}$ lines in vibrational states below $2 \nu_{7}$ show an emission that exceeds what is predicted by the SEE model. Including the CCS component as in $\S 4.3$ provides a first approximation to the detected signal (see Fig. 5) that is in fact quite good for $J_{\text {up }}$ below $\sim 20$. However, the HVW component is also visible in the high- $J v=0$ lines and therefore should be included in the model. The high-velocity outflow is modeled by just considering a gas component with a given excitation temperature $(350 \mathrm{~K})$, average column density $\left(2.5 \times 10^{17} \mathrm{~cm}^{-2}\right)$, filling factor at a reference frequency $\left(1.3 \times 10^{-3}\right.$ at $\left.90 \mathrm{GHz}\right)$, and half-width outflow velocity $\left(150 \mathrm{~km} \mathrm{~s}^{-1}\right)$. The rather high temperature for this component is confirmed by the dominant role that the broad HVW component has at high $J$ values (see CSO lines in Fig. 5). More precise details about the structure of the fast outflow are difficult to obtain with the present data. A

TABLE 2

Model Parameters of the Circumstellar Shell (See Fig. 3) That Provide the Best Fit to the $\mathrm{HC}_{5} \mathrm{~N}$ Rotational Lines in the Ground Vibrational State (See Fig. 4)

\begin{tabular}{|c|c|}
\hline Parameter & Value \\
\hline $2 R_{\mathrm{CCS} 1}(\operatorname{arcsec}) \ldots \ldots$ & 3.0 \\
\hline $2 R_{\mathrm{CCS} 2}(\operatorname{arcsec}) \ldots \ldots \ldots \ldots \ldots \ldots \ldots \ldots$ & 4.5 \\
\hline$\theta_{d}(\mathrm{deg}) \ldots \ldots \ldots \ldots \ldots \ldots \ldots \ldots \ldots \ldots \ldots$ & 40 \\
\hline$\alpha_{T}(\operatorname{deg}) \ldots \ldots \ldots \ldots \ldots \ldots$ & 30 \\
\hline$T_{\mathrm{rot}, \mathrm{HC}}^{\mathrm{CCS}}(\mathrm{K})$ & $50-70$ \\
\hline 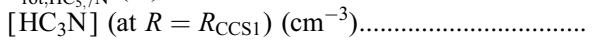 & 0.8 \\
\hline$d_{\mathrm{CCS}}($ no units) & -1.0 \\
\hline$\rho_{\mathrm{CCS}}$ (no units) ...... & $1 / 4$ \\
\hline$\left[\mathrm{HC}_{3} \mathrm{~N}\right] /\left[\mathrm{HC}_{5} \mathrm{~N}\right] \ldots$. & 3 \\
\hline$\left[\mathrm{HC}_{5} \mathrm{~N}\right] /\left[\mathrm{HC}_{7} \mathrm{~N}\right] \ldots \ldots \ldots \ldots$ & 6 \\
\hline$v_{\mathrm{CCS}}\left(\right.$ at $\left.R=R_{\mathrm{CCS} 1}\right)\left(\mathrm{km} \mathrm{s}^{-1}\right) \ldots \ldots \ldots$ & 22 \\
\hline 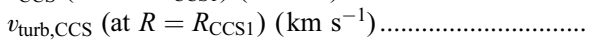 & 12 \\
\hline 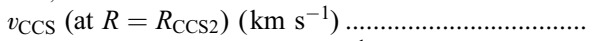 & 8 \\
\hline 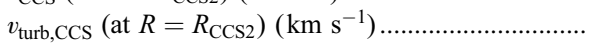 & 5 \\
\hline
\end{tabular}

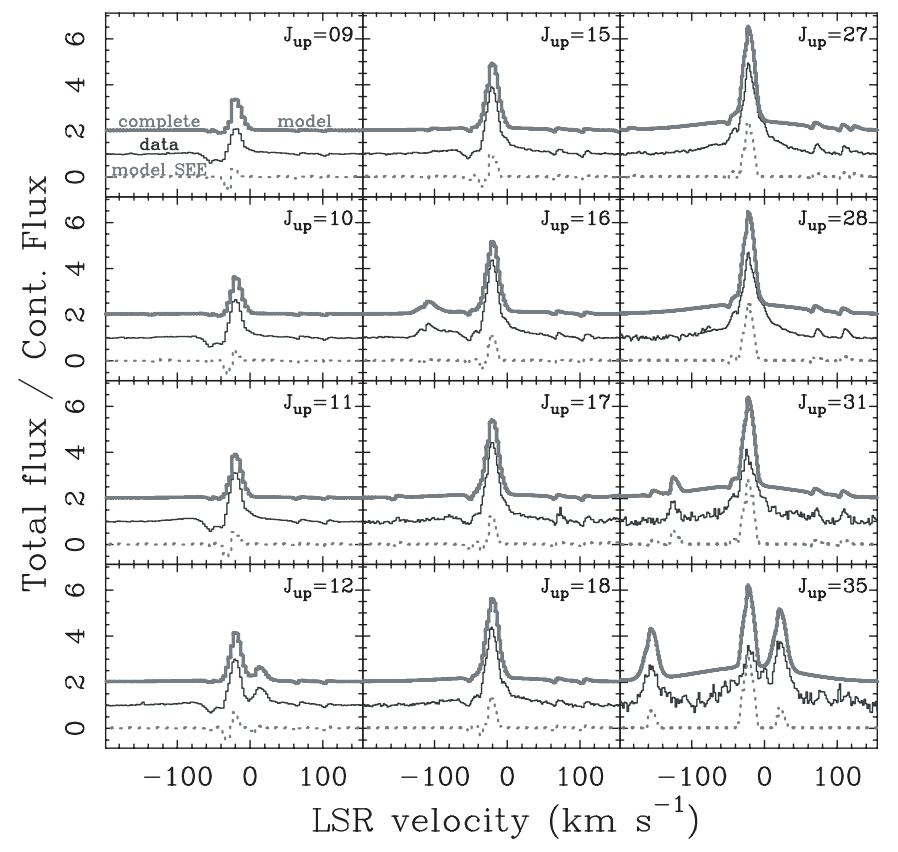

FIG. 5.-Observed rotational lines of $\mathrm{HC}_{3} \mathrm{~N}$ in the ground vibrational state compared with the $\mathrm{SEE}+\mathrm{CCS}$ model assuming a $\mathrm{HC}_{3} \mathrm{~N} / \mathrm{HC}_{5} \mathrm{~N}$ value of 3 , plus a HVW component according to $\S 4.5$ (solid histogram, shifted by $1.0 F_{c}$ ). The model for the SEE region alone is also plotted for comparison (dotted line, shifted by $-1.0 F_{c}$ ). Some other lines appear in the data and are also modeled. Most of them are rotational transitions within different vibrational states of $\mathrm{HC}_{3} \mathrm{~N}, \mathrm{HC}_{5} \mathrm{~N}$, or their isotopologues, but there are some belonging to other species, the discussion of which we leave for a separate work: $\mathrm{H}_{2} \mathrm{CO}$ and $\mathrm{C}_{5} \mathrm{H}$ in $J_{\text {up }}=16$ and c- $\mathrm{C}_{3} \mathrm{H}_{2}$ in $J_{\text {up }}=35$. All observations have been carried out with the IRAM $30 \mathrm{~m}$ telescope, except $J_{\text {up }}=31$ and 35 (CSO). Velocity resolutions of the data and model are the same, as explained in previous figures. 


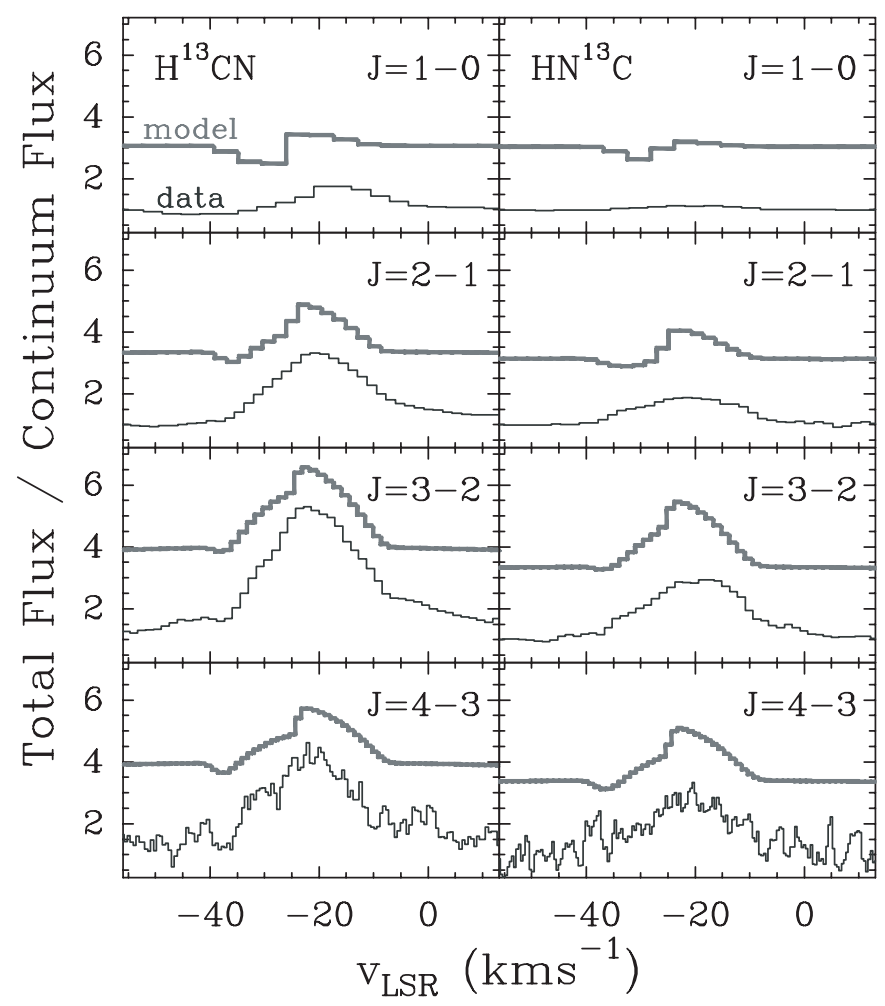

FIG. 6.- Observed rotational lines of $\mathrm{H}^{13} \mathrm{CN}$ and $\mathrm{HN}^{13} \mathrm{C}$ compared with the $\mathrm{SEE}+\mathrm{HVW}$ model (shifted by $2.0 F_{c}$ ) assuming $\mathrm{H}^{13} \mathrm{CN} / \mathrm{HC}_{3} \mathrm{~N}$ and $\mathrm{HN}^{13} \mathrm{C} /$ $\mathrm{HC}_{3} \mathrm{~N}$ values of $1 / 2$ and $2 / 15$, respectively. All observations have been carried out with the IRAM $30 \mathrm{~m}$ telescope, except $J_{\text {up }}=4$ (CSO). The very low excitation temperatures of the $J_{\text {up }}=1$ and 2 levels, compared to those in the SEE and HVW regions, results in the poor agreement between the data and model for the lines arising from those levels. Nevertheless, the other lines provide a useful upper limit (the CCS contribution should be important) to the HCN and HNC abundances in the SEE and HVW regions. Velocity resolutions of the model and data are the same for the different lines, as explained in previous figures.

better constraint on the temperature should be obtained by observing a few $v=0 \mathrm{HC}_{3} \mathrm{~N}$ lines above $400 \mathrm{GHz}$ (ongoing).

\section{6. $\mathrm{HCN}$ and $\mathrm{HNC}$ Emission}

At this point of the analysis we have a model that considers the three main regions of the gas emission in the millimeter wave spectrum of CRL 618: SEE, CCS, and HVW. We can try to fit the observed lines from other species by adjusting only their ratio to $\mathrm{HC}_{3} \mathrm{~N}$ with the assumption that the rotational temperature is the same. This approximation will be quite good for those species arising mainly from the SEE, due to the high density of the region (populations are thermalized). It could also be useful for the HVW. However, the low densities imply that the full analysis for all molecular species will be more complex in the CCS.

In this work we focus only on $\mathrm{HCN}$ and $\mathrm{HNC}$. For opacity reasons, we cannot probe the inner SEE and HVW regions using the main isotopologues of both species, especially as $J$ increases. Instead, we can use $\mathrm{H}^{13} \mathrm{CN}$ and $\mathrm{HN}^{13} \mathrm{C}$ assuming a $\mathrm{SEE}{ }^{12} \mathrm{C} /{ }^{13} \mathrm{C}$ value of 15 (see above), but even for these isotopologues some contribution from the CCS can be expected. As a result, our simulations considering only the SEE + HVW (see Fig. 6) for $J_{\text {up }}>2$ provide upper limits for the $\mathrm{H}^{13} \mathrm{CN} /$ $\mathrm{HC}_{3} \mathrm{~N}$ and $\mathrm{HN}^{13} \mathrm{C} / \mathrm{HC}_{3} \mathrm{~N}$ ratios in the SEE. The values obtained are about $1 / 2$ and $2 / 15$, respectively, which translate into $\mathrm{HCN} / \mathrm{HC}_{3} \mathrm{~N} \sim 7.5$ and $\mathrm{HCN} / \mathrm{HC}_{3} \mathrm{~N} \sim 2.0$.

To improve the above estimates in the SEE, it is better to use vibrationally excited states of $\mathrm{HCN}$ and $\mathrm{HNC}$, since the $\mathrm{CCS}$

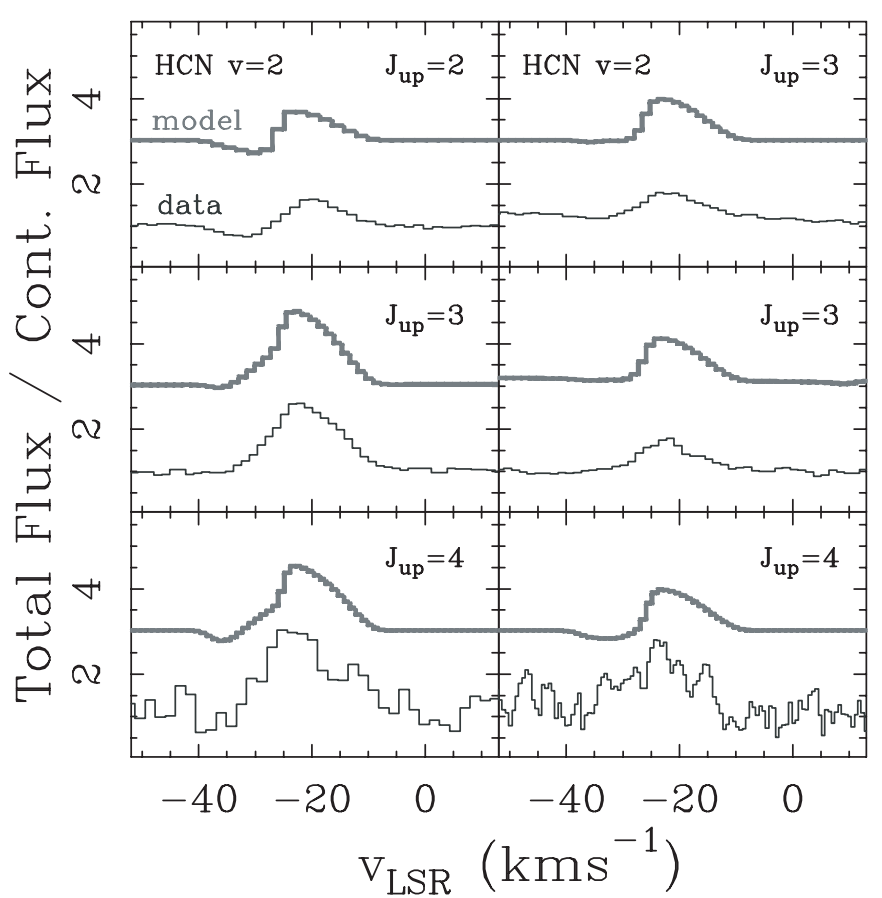

FIG. 7.- Observed rotational lines of $\mathrm{HCN}$ and $\mathrm{HNC}$ in the $v=2$ vibrational state compared with the SEE model (shifted by $2.0 F_{c}$ ) assuming $\mathrm{HCN} / \mathrm{HC}_{3} \mathrm{~N}$ and $\mathrm{HNC} / \mathrm{HC}_{3} \mathrm{~N}$ values of 2 and $1 / 5$, respectively. All observations have been carried out with the IRAM $30 \mathrm{~m}$ telescope, except $J_{\text {up }}=4$ (CSO). Velocity resolutions of the data and model are the same, as in previous figures.

contribution should be negligible for them. The lowest one $\left(v_{2}=1\right)$, at 712 and $477 \mathrm{~cm}^{-1}$ for $\mathrm{HCN}$ and HNC, respectively, is detected in both molecules ( $l$ doublets for all rotational transitions except the forbidden $J=1-0)$. We have selected those lines that have been observed and are not badly blended with other species to get a better constraint for the above ratios. This analysis (Fig. 7) results in $\mathrm{HCN} / \mathrm{HC}_{3} \mathrm{~N} \sim 2$ and $\mathrm{HNC} / \mathrm{HC}_{3} \mathrm{~N} \sim 1 / 3-1 / 5$. It is thus demonstrated that much lower ratios of $\mathrm{HCN}$ and $\mathrm{HNC}$ with respect to longer cyanopolyynes are found in the inner gas of CRL 618 (PPN phase) compared to those found in IRC +10216 (AGB phase). This is discussed in $\S 5$.

\subsection{Ground-State $\mathrm{HC}_{7} \mathrm{~N}$}

Finally, we have studied the $\mathrm{HC}_{7} \mathrm{~N}$ rotational lines within the $v=0$ state. This molecule is clearly detected, since all transitions between $J_{\text {up }}=72$ and $J_{\text {up }}=85$ appear above a $3 \sigma$ level, except for three cases due to a coincidence with a much stronger line (see Table 3 and Fig. 8). Note that most of the $\mathrm{HC}_{7} \mathrm{~N} v=0$ signal arises from the CCS. We reach this conclusion after running models for which no $\mathrm{HC}_{7} \mathrm{~N}$ was present in the CCS and its ratio to $\mathrm{HC}_{5} \mathrm{~N}$ was set to be $1 / 3$ in the $\mathrm{SEE}$. In this case, the model predicts some detectable absorption and almost no noticeable emission. The fact that $\mathrm{HC}_{7} \mathrm{~N}$ is seen almost exclusively in emission with a much larger intensity than in this model can be easily reproduced if the CCS is included with a $\mathrm{HC}_{5} \mathrm{~N} / \mathrm{HC}_{7} \mathrm{~N}$ abundance ratio around 3 and the same rotational temperature for both species (see Fig. 8). The model also predicts that above $J_{\text {up }}=85$ (at an energy level of $134 \mathrm{~cm}^{-1}$ ) the signal would be too weak to be detected above the $3 \sigma$ level of the survey. Similarly, the intensity of lines of $\mathrm{HC}_{9} \mathrm{~N}$, with a ratio of $1 / 3$ or less with respect to $\mathrm{HC}_{7} \mathrm{~N}$, is estimated to be well below the detection limit of our observations. 
TABLE 3

Detected Lines of $\mathrm{HC}_{7} \mathrm{~N}$ toward CRL 618

\begin{tabular}{|c|c|c|c|}
\hline \multirow[b]{2}{*}{$J_{\text {up }}$} & \multicolumn{3}{|c|}{ VibRational State of $\mathrm{HC}_{7} \mathrm{~N}$} \\
\hline & $v=0$ & $\nu_{15} 1^{-}$ & $\nu_{15} 1^{+}$ \\
\hline 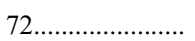 & 81.2101 & $(81.3115)$ & $(81.3630)$ \\
\hline 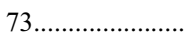 & 82.3379 & $\ldots$ & (82.4929) \\
\hline $74 \ldots \ldots \ldots \ldots \ldots \ldots \ldots . .$. & 83.4656 & $\ldots$ & $\ldots$ \\
\hline $75 \ldots \ldots \ldots \ldots \ldots \ldots \ldots$ & 84.5933 & (84.6989) & $\ldots$ \\
\hline $76 \ldots \ldots \ldots \ldots \ldots \ldots \ldots \ldots$ & 85.7211 & $(85.8281)$ & $(85.8824)$ \\
\hline $77 \ldots \ldots \ldots \ldots \ldots \ldots \ldots \ldots$ & & $(86.9572)$ & $(87.0122)$ \\
\hline $78 \ldots \ldots \ldots \ldots \ldots \ldots \ldots \ldots$ & 87.9765 & & $(88.1421)$ \\
\hline 79.......................... & 89.1042 & $(89.2154)$ & (89.2719) \\
\hline 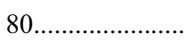 & $\ldots$ & $\ldots$ & $\ldots$ \\
\hline 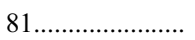 & 91.3596 & $\ldots$ & $\ldots$ \\
\hline 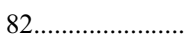 & 92.4873 & $\ldots$ & $\ldots$ \\
\hline 83 & $\ldots$ & $\cdots$ & $\ldots$ \\
\hline 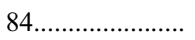 & 94.7426 & $\ldots$ & $\ldots$ \\
\hline 85................................. & 95.8703 & $\ldots$ & $\ldots$ \\
\hline 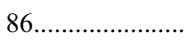 & $\ldots$ & $\ldots$ & $\ldots$ \\
\hline 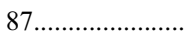 & $\ldots$ & $\ldots$ & $\ldots$ \\
\hline $88 \ldots \ldots \ldots \ldots \ldots \ldots \ldots$ & $\ldots$ & $\ldots$ & $\ldots$ \\
\hline
\end{tabular}

Notes.- Laboratory frequencies in GHz are rounded to $0.1 \mathrm{MHz}$. Frequencies in parentheses correspond to co-added lines in the velocity scale for a tentative detection of $\nu_{15}$ (see text).

\subsection{Vibrationally Excited $\mathrm{HC}_{7} \mathrm{~N}$}

The frequencies of the rotational transitions in the lowest energy bending modes of $\mathrm{HC}_{7} \mathrm{~N}\left(\nu_{13}, \nu_{14}\right.$, and $\left.\nu_{15}\right)$ have become available in a recent work by Bizzochi \& Degli Esposti (2004). We have searched for those in the lowest vibrational state $(0,0,1)$, but no individual detection has been obtained. These lines have been modeled by considering computed ab initio values for the $(0,0,1),(0,1,0)$, and $(1,0,0)$ energies: 62,163 , and $280 \mathrm{~cm}^{-1}$, respectively (Botschwina et al. 1997). A tentative detection is obtained when performing the addition in the $v_{\text {LSR }}$ scale of the observed signal at the positions of the $1^{-}$and $1^{+} J_{\text {up }}=72-79$ lines (see Fig. 8). Five lines in the considered $J_{\text {up }}$ range were discarded from the summation because of blending with stronger lines (see Table 3).

\section{COMPARISON WITH CHEMICAL MODELS}

The abundance ratio for consecutive members of the cyanopolyyne family of molecules in IRC +10216 is $\sim 3$ (Cernicharo et al. 1987). However, the $\mathrm{HCN} / \mathrm{HC}_{3} \mathrm{~N}$ ratio in the same object is larger than 100. In CRL 618 our results indicate also an abundance ratio near 3 for $\mathrm{HC}_{3} \mathrm{~N} / \mathrm{HC}_{5} \mathrm{~N}$ and $\sim 6$ for $\mathrm{HC}_{5} \mathrm{~N} /$ $\mathrm{HC}_{7} \mathrm{~N}$, but for $\mathrm{HCN} / \mathrm{HC}_{3} \mathrm{~N}$ it is around 2-3. The difference in this ratio at different evolutionary stages of C-rich objects tells us about the chemical processing of the gas when the central star evolves toward the white dwarf stage. Cernicharo (2004) has modeled the role of the strong UV field arising from the hot central star in the chemical abundances of the polyacetylenes, cyanopolyynes, and carbon clusters. This work shows that $\mathrm{HCN}$ is quickly photodissociated, allowing the production of $\mathrm{CN}$, which reacts with $\mathrm{C}_{2} \mathrm{H}_{2}$ and $\mathrm{C}_{2} \mathrm{H}$ to produce $\mathrm{HC}_{3} \mathrm{~N}$ and longer cyanopolyynes, thus explaining the low $\mathrm{HCN} / \mathrm{HC}_{3} \mathrm{~N}$ ratio found in CRL 618. Moreover, this behavior is similar to that found by Cernicharo et al. (2001) for $\mathrm{C}_{4} \mathrm{H}_{2}$ and $\mathrm{C}_{6} \mathrm{H}_{2}$. The large abundance of $\mathrm{HNC}$ with respect to $\mathrm{HCN}$ in $\mathrm{CRL} 618$ (HCN/HNC $\sim 10$ instead of $>200$ in IRC +10216 ; Cernicharo et al. 1987) also points in the same direction. It seems that in a short period of time

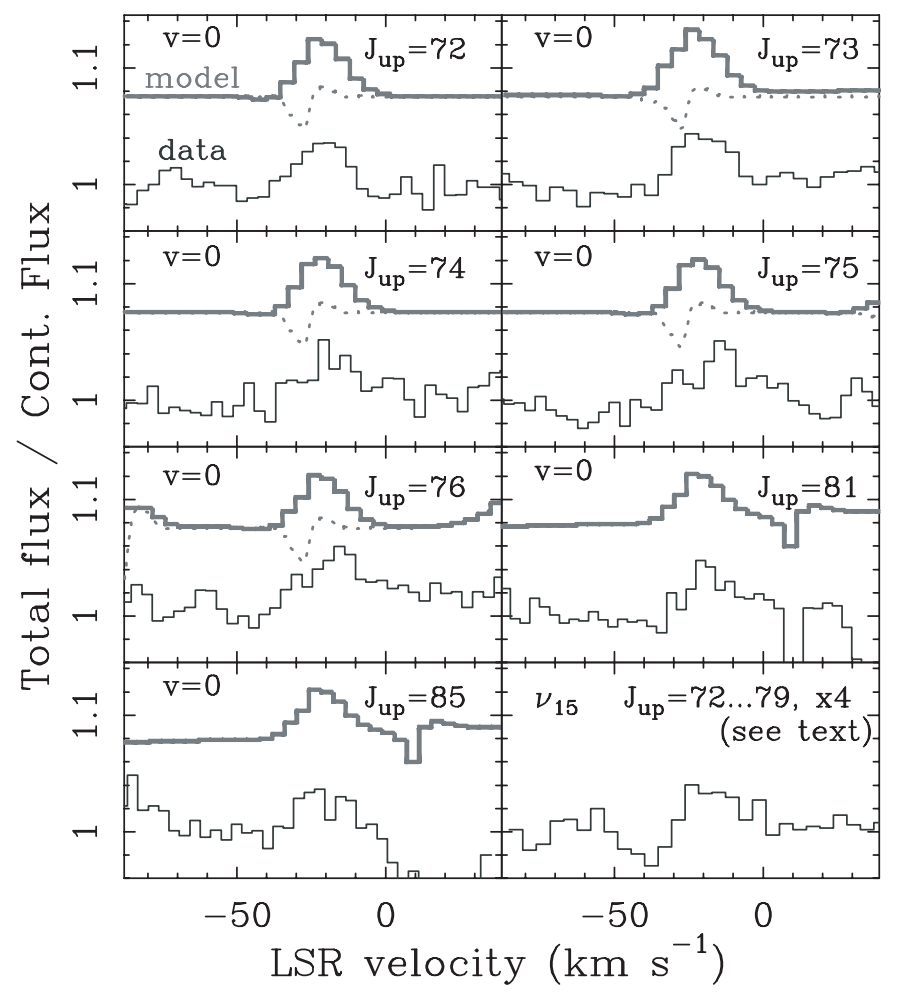

FIG. 8. $-\mathrm{HC}_{7} \mathrm{~N}(v=0)$ lines observed toward CRL 618 compared with model results including the SEE and CCS regions (solid histogram, shifted by $0.075 F_{c}$ ) and the CCS region alone (dotted line, also shifted by $0.075 F_{c}$ ). The considered value of $\mathrm{HC}_{5} \mathrm{~N} / \mathrm{HC}_{7} \mathrm{~N}$ is 3 , which provides a very good fit. The spectrum in the bottom right panel is the sum of the $\nu_{15} \mathrm{HC}_{7} \mathrm{~N}$ rotational lines listed in Table 3 . A 4.0 scaling factor has been introduced to keep the same scale as all other spectra.

it could be possible to transform an important fraction of $\mathrm{HCN}$ into $\mathrm{HC}_{3} \mathrm{~N}$ and longer cyanide carbon chains.

\section{SUMMARY AND FUTURE WORK}

We have extended our previous analysis of the gas shells surrounding the protoplanetary nebula CRL 618 by studying the pure rotational lines of $\mathrm{HC}_{5} \mathrm{~N}$ in its fundamental and the lowest four vibrationally excited states (the first astronomical source in which vibrationally excited $\mathrm{HC}_{5} \mathrm{~N}$ has been detected) and $\mathrm{HC}_{7} \mathrm{~N}$ rotational lines in its fundamental vibrational state. We have found that the $\mathrm{HC}_{3} \mathrm{~N} / \mathrm{HC}_{5} \mathrm{~N}$ ratio in the innermost slowly expanding envelope is $\sim 3$. With this ratio, and all the physical parameters previously derived in $\mathrm{P} 04$, a good match to the observed $\mathrm{HC}_{5} \mathrm{~N}$ lines in vibrationally excited states is found. However, the predicted lines for the ground vibrational state are far too weak, suggesting that a more extended and colder gas shell has to be considered. The physical parameters of this envelope, called the cold circumstellar shell (CCS) in this work, have been determined. The shell extends between diameters 3."0 and 4.5 , and its excitation temperature is only $\sim 60 \mathrm{~K}$ (compared to $250-275 \mathrm{~K}$ in the innermost SEE). The column density of the CCS in front of the continuum source is negligible compared to that of the SEE. With the CCS derived parameters it is found that the observed $\mathrm{HC}_{7} \mathrm{~N}$ lines in the ground vibrational state can be well reproduced with a $\mathrm{HC}_{5} \mathrm{~N} / \mathrm{HC}_{7} \mathrm{~N}$ abundance ratio of $\sim 3$. The isotopic ratio ${ }^{12} \mathrm{C} /{ }^{13} \mathrm{C}$ is found to be $\sim 15$ in the SEE from the lines of vibrationally excited $\mathrm{HC}_{3} \mathrm{~N}$. The same value must be an upper limit in the CCS, according to the nondetection of ${ }^{13} \mathrm{C}$ substituted isotopologues of $\mathrm{HC}_{5} \mathrm{~N}$. 
The analysis of the $v=0 \mathrm{HC}_{3} \mathrm{~N}$ lines requires introducing a third component, the high-velocity outflow, although its structure cannot be precisely known. Finally, the ratios HCN/ $\mathrm{HC}_{3} \mathrm{~N}$ and $\mathrm{HNC} / \mathrm{HC}_{3} \mathrm{~N}$ have been derived using the first four rotational transitions of $\mathrm{H}^{13} \mathrm{CN}$ and $\mathrm{HN}^{13} \mathrm{C}$ and some lines of vibrationally excited $\mathrm{HCN}$. The result of a much lower $\mathrm{HCN}$ / $\mathrm{HC}_{3} \mathrm{~N}$ value than in IRC +10216 indicates a processing of $\mathrm{HCN}$ into longer cyanopolyynes in the protoplanetary nebula stage.

A first examination of lines from all other molecules detected in the survey shows that most of them can be well fitted using the model of the CCS, HVW, and SEE gas regions described in this paper and in P04, so that a good estimate of their abun- dances in these regions can be achieved. Therefore, the next step in our analysis of the CRL 618 line survey will be to provide a picture of the chemical content in its three main gas components.

We acknowledge the support of the IRAM $30 \mathrm{~m}$ staff during the long completion of the line survey. CSO operations are supported by US NSF grant AST 22-09008. This work has also been supported by Spanish DGES and PNIE grants ESP 2002-01627, AYA 2002-10113-E, and AYA 2003-02785-E.
Bizzocchi, L., \& Degli Esposti, C. 2004, ApJ, 614, 518

Botschwina, P., Horn, M., Markey, K., \& Oswald, R. 1997, Mol. Phys., 92, 381

Bujarrabal, V., Gomez-Gonzalez, J., Bachiller, R., \& Martin-Pintado, J. 1988, A\&A, 204, 242

Cernicharo, J. 2004, ApJ, 608, L41

Cernicharo, J., Guélin, M., Martin-Pintado, J., Peñalver, J., \& Mauersberger, M. 1989, A\&A, 222, L1 (C89)

Cernicharo, J., Guélin, M., Menten, K. M., \& Walmsley, C. M. 1987, A\&A, 181, L1

Cernicharo, J., Heras, A. M., Tielens, A. G. G. M., Pardo, J. R., Herpin, F., Guélin, M., \& Waters, L. B. F. M. 2001, ApJ, 546, L123

Hajian, A. R., Phillips, J. A., \& Terzian, Y. 1995, ApJ, 446, 244

Herpin, F., Goicoechea, J. R., Pardo, J. R., \& Cernicharo, J. 2002, ApJ, 577, 961

Kahane, C., Cernicharo, J., Gómez-González, J., \& Guélin, M. 1992, A\&A, 256,235

\section{REFERENCES}

Kroto, H. W., Kirby, C., Walton, D. R. M., Avery, L. W., Broten, N. W., MacLeod, J. M., \& Oka, T. 1978, ApJ, 219, L133

Little, L. T., MacDonald, G. H., Riley, P. W., \& Matheson, D. N. 1978, MNRAS, 183, 45P

Neri, R., Garcia-Burillo, S., Guélin, M., Cernicharo, J., Guilloteau, S., \& Lucas, R. 1992, A\&A, 262, 544

Pardo, J. R., Cernicharo, J., Goicoechea, J. R., \& Phillips, T. G. 2004, ApJ, 615, 495 (P04)

Sánchez-Contreras, C., \& Sahai, R. 2004, ApJ, 602, 960

Snell, R. L., Schloerb, F. P., Young, J. S., Hjalmarson, A., \& Friberg, P. 1981, ApJ, 244, 45

Yamada, K. M. T., Degli Esposti, C., Botschwina, P., Förster, P., Bizzocchi, L., Thorwirth, S., \& Winnewisser, G. 2004, A\&A, 425, 767 